\title{
Antibiotic resistance in isolates recovered from women with community-acquired urinary tract infections presenting to a tertiary care emergency department
}

\author{
Lyne Filiatrault, MD** ${ }^{\dagger}$; Rachel M. McKay, MSc ${ }^{\ddagger}$; David M. Patrick, MD, MHSc ${ }^{\ddagger \S}$; \\ Diane L. Roscoe, MD"\#; Grahame Quan, BSc, BA**; Jeff Brubacher, MD**; Ken M. Collins, BSc ${ }^{\dagger \dagger}$
}

\section{ABSTRACT}

Introduction: We sought to determine the antibiotic susceptibility of organisms causing community-acquired urinary tract infections (UTIs) in adult females attending an urban emergency department (ED) and to identify risk factors for antibiotic resistance.

Methods: We reviewed the ED charts of all nonpregnant, nonlactating adult females with positive urine cultures for 2008 and recorded demographics, diagnosis, complicating factors, organism susceptibility, and risk factors for antibiotic resistance. Odds ratios (ORs) and 95\% confidence intervals (Cls) for potential risk factors were calculated.

Results: Our final sample comprised 327 UTIs: 218 were cystitis, of which 22 were complicated cases and 109 were pyelonephritis, including 22 complicated cases. Escherichia coli accounted for $82.3 \%$ of all UTIs, whereas Staphylococcus saprophyticus accounted for $5.2 \%$. In uncomplicated cystitis, $9.5 \%$ of all isolates were resistant to ciprofloxacin and $24.0 \%$ to trimethoprim-sulfamethoxazole (TMP-SMX). In uncomplicated pyelonephritis, $19.5 \%$ of isolates were resistant to ciprofloxacin and $36.8 \%$ to TMP-SMX. In UTI (all types combined), any antibiotic use within the previous 3 months was a significant risk factor for resistance to both ciprofloxacin (OR 3.34, 95\% Cl 1.16-9.62) and TMP-SMX (OR 4.02, 95\% $\mathrm{Cl}$ 1.48-10.92). Being 65 years of age or older and having had a history of UTI in the previous year were risk factors only for ciprofloxacin resistance.

Conclusions: $E$. coli was the predominant urinary pathogen in this series. Resistance to ciprofloxacin and TMP-SMX was high, highlighting the importance of relevant, local antibiograms. Any recent antibiotic use was a risk factor for both ciprofloxacin and TMP-SMX resistance in UTI. Our findings should be confirmed with a larger prospective study.

\section{RÉSUMÉ}

Introduction: L'étude visait à déterminer la sensibilité aux antibiotiques des micro-organismes causant des infections urinaires (IU) extrahospitalières, chez des femmes adultes, vues à un service urbain des urgences, ainsi qu'à cerner des facteurs de risque de résistance aux antibiotiques.

Méthodes: Nous avons passé en revue les dossiers de toutes les femmes adultes, non enceintes et non allaitantes, vues à I'urgence en 2008 et ayant obtenu une uroculture positive, en plus de consigner les données démographiques, le diagnostic, les facteurs de complication, la sensibilité des microorganismes, et les facteurs de risque de résistance aux antibiotiques. Nous avons calculé le risque relatif approché (RRA) et l'intervalle de confiance (IC) à $95 \%$ des facteurs de risque possibles.

Résultats: L'échantillon définitif totalisait 327 IU: 218 cystites, dont 22 avec complications, et 109 pyélonéphrites, dont 22 avec complications. Escherichia coli était responsable de $82.3 \%$ des IU, tandis que Staphylococcus saprophyticus en représentait $5.2 \%$. Dans la cystite sans complications, $9.5 \%$ des isolats étaient résistants à la ciprofloxacine et $24.0 \%$ au triméthoprime-sulfaméthoxazole (TMP/SMX). Dans la pyélonéphrite sans complications, $19.5 \%$ des isolats étaient résistants à la ciprofloxacine et $36.8 \%$ au TMP/SMX. Dans les IU, tous types confondus, I'utilisation d'antibiotiques au cours des 3 mois précédents s'est révélée un facteur de risque important de résistance à la ciprofloxacine (RRA 3.34; IC à 95\% 1.169.62) et au TMP/SMX (RRA 4.02; IC à 95\% 1.48-10.92). Par ailleurs, le fait d'être âgé d'au moins 65 ans et d'avoir eu des antécédents d'IU au cours de l'année précédente se sont révélés des facteurs de risque de résistance à la ciprofloxacine seulement.

From the *§\#* Department of Emergency Medicine, School of Population and Public Health, Department of Pathology and Laboratory Medicine, and School of Medicine, University of British Columbia, Vancouver, BC; $+\|$ Emergency Department and Division of Medical Microbiology and Infection Control, Vancouver General Hospital; †BC Centre for Disease Control, Vancouver, BC; †tSchool of Medicine, Queen's University, Kingston, ON.

Correspondence to: Dr. Lyne Filiatrault, VGH Emergency Department, 855 West 12th Avenue, Vancouver, BC V5Z IM9; filiatra@interchange.ubc. ca.

This article has been peer reviewed. 
Conclusions: Dans la présente série, $E$. coli était le principal agent pathogène des voies urinaires. La fréquence de la résistance à la ciprofloxacine et au TMP/SMX était élevée, d'où l'importance des antibiogrammes locaux, pertinents. Toute utilisation récente $d^{\prime}$ antibiotiques s'est révélée un facteur de risque de résistance à la ciprofloxacine et au TMP-SMX dans les IU. II faudrait confirmer les résultats de la recherche dans le cadre d'une étude prospective, menée à grande échelle.

Keywords: antibiotic resistance, community acquired, risk factors, urinary tract infection
After respiratory tract infections, urinary tract infections (UTIs) are the most common infections for which patients seek medical attention in the community. ${ }^{1}$ In 2000, there were 6.3 million office visits and 1.3 million emergency department (ED) visits for UTIs in women in the United States. ${ }^{2}$ An estimated $20 \%$ of women between 20 and 65 years will experience one UTI every year, ${ }^{3}$ and $50 \%$ of women have at least one UTI in their lifetime. ${ }^{4}$ Recurrences occur within 1 year in $48 \%$ of women. ${ }^{5}$

Treatment of a UTI with an antibiotic to which the organism is resistant results in high rates of microbiologic and clinical failure and leads to additional morbidity and costs. ${ }^{6-9}$ Resistance rates vary geographically, ${ }^{10,11}$ with the population studied, and according to whether or not the UTI is complicated ${ }^{12-14}$ (see Table 1 and Table 2 for definitions). Hospital laboratory surveillance studies have limited applicability to the ED outpatient population with community-acquired UTIs because young, healthy, premenopausal women with uncomplicated cystitis are typically treated empirically without urine cultures. ${ }^{12}$ The hospital antibiogram-local susceptibility results of isolated bacterial strains based on laboratory testing - is based on urine cultures from patients with more severe infections, complicated UTIs, recurrent infections, or treatment failures and thus overestimates the prevalence of antibiotic resistance in the community..$^{13,15,16}$

Our primary goal was to describe the organisms responsible for community-acquired cystitis and pyelonephritis (PN) in the adult nonpregnant female ED population and their susceptibility to commonly prescribed antibiotics. Our secondary goal was to identify risk factors for antibiotic resistance in uncomplicated and complicated cystitis and PN. Visits (not admissions) to Vancouver General Hospital (VGH) within the last 4 weeks, antibiotic use within the last 3 months, and positive urine cultures in the previous year were investigated as potential risk factors for ciprofloxacinresistant uropathogens, following the results of the VGH ED urosepsis study (personal communication, C Lowe, 2009). We also investigated age as a potential risk factor.

\section{METHODS}

\section{Study setting}

This study was conducted at VGH, an 800-bed tertiary care centre in Vancouver, British Columbia, with an annual ED census of 75,000 adult patients and an admission rate of $23 \%$.

\section{Ethics}

This study was approved by the University of British Columbia's Clinical Research Ethics Board.

\section{Definitions}

UTIs can be either cystitis or PN (see Table 1 for definitions), and each of these can be considered uncomplicated or complicated, depending on the presence or absence of certain factors (see Table 2), based on the literature. ${ }^{12,14,17}$ Catheter samples were obtained from patients with complicated UTIs with a pre-existing indwelling catheter. In uncomplicated UTIs, samples were clean-catch midstream urine collection, with the rare in-and-out catheter sample.

\begin{tabular}{|c|c|}
\hline Term & Defined as \\
\hline Positive urine culture & $\begin{array}{l}\text { Growing one or two } \\
\text { uropathogens, either one } \geq \\
10^{4} \mathrm{CFU} / \mathrm{mL}\left(\geq 10^{7} \mathrm{CFU} / \mathrm{L}\right)\end{array}$ \\
\hline $\begin{array}{l}\text { Cystitis (infection of lower } \\
\text { urinary tract or bladder) }\end{array}$ & $\begin{array}{l}\text { Positive urine culture result as } \\
\text { per above } \\
\text { and } \\
\text { One or more of dysuria, } \\
\text { frequency, urgency, and/or } \\
\text { hematuria }\end{array}$ \\
\hline $\begin{array}{l}\text { Pyelonephritis (infection of } \\
\text { upper urinary tract) }\end{array}$ & $\begin{array}{l}\text { Positive urine culture result } \\
\text { and } \\
\text { Flank pain and/or costovertebral } \\
\text { angle tenderness; presence of a } \\
\text { documented fever was not } \\
\text { necessary }\end{array}$ \\
\hline
\end{tabular}




\begin{tabular}{l} 
Table 2. Complicating factors \\
Anatomic/functional \\
Solitary kidney \\
Polycystic kidneys \\
Renal abscess \\
Duplicated collecting system \\
Instrumentation \\
Indwelling or intermittent catherization \\
Ureteric stent \\
Nephrostomy tube \\
Recent cystoscopy \\
Vesicoureteral reflux \\
Surgical reconstruction \\
Illeal conduit \\
Bladder augmentation \\
Renal transplant \\
Neurogenic bladder \\
Urinary incontinence \\
Obstruction \\
Stone \\
Tumour \\
Stricture \\
Medical condition \\
Diabetes mellitus \\
Renal failure \\
Liver failure \\
Immunosuppression \\
Corticosteroid use \\
Human immunodeficiency virus (HIV) \\
Active cancer \\
\hline
\end{tabular}

\section{Inclusion and exclusion criteria}

We included adult ( $\geq 19$ years) nonpregnant, nonlactating females who presented to the ED in 2008 with signs and symptoms consistent with cystitis or $\mathrm{PN}$ and who had a positive urine culture. We excluded long-term care or nursing home residents as well as patients who had been admitted to VGH or Vancouver Coastal Health Authority-affiliated hospitals (Richmond Hospital and University of British Columbia Hospital) in the previous 4 weeks. Patients with asymptomatic bacteriuria were excluded, as were patients with urosepsis. Patients were enrolled only once.

\section{Data sources}

We accessed the hospital microbiology and ED visit databases to identify all adult female ED patients with positive urine cultures (January 1 to December 31, 2008) who were discharged from the ED or admitted to hospital with a diagnosis of cystitis or PN (Appendix 1).

\section{Data collection}

Data were collected in accordance with recommendations for medical record reviews in emergency medicine. ${ }^{18,19}$ Two trained investigators determined eligibility and entered data from the medical record on a computer-based spreadsheet (Appendix 2). We used explicit definitions for each data category. The abstraction process was monitored by the first author. Coding uncertainties were resolved in weekly meetings. A random sample of 57 medical records was reviewed by both reviewers to determine interobserver agreement using the kappa ( $\kappa)$ statistic.

\section{Outcome measures}

Antimicrobial susceptibility testing was performed according to Clinical and Laboratory Standards Institute (CLSI) procedures using the standardized disk diffusion or commercially available automated microdilution methods. Organisms were classified as "susceptible," "intermediate," or "resistant" to each of the antibiotics tested based on zones of inhibition (disk diffusion) or minimum inhibitory concentrations (automated microdilution methods). Applying the most conservative clinical interpretation, we defined "antibiotic resistance" as including both intermediate and resistant sensitivities. Any reference to resistance in the remainder of this article includes intermediate unless otherwise indicated. We excluded cultures growing more than two organisms (considered contaminated) and cultures with nonuropathogens.

\section{Statistical analysis}

Data were analyzed using SAS 9.2 for Windows (SAS Institute, Cary, NC) and SPSS 14 for Windows (SPSS Inc, Chicago, IL). We used the Mann-Whitney $U$ test to compare for differences in continuous variables and the chi-square test or Fisher exact test, as appropriate, for categorical variables. Odds ratios (ORs) and 95\% confidence intervals (CIs) were calculated. Statistical significance was set at $\alpha=0.05$.

\section{RESULTS}

\section{Patients' demographics and documented risk factors prevalence}

We identified 439 female ED patients with positive urine cultures and a diagnosis of cystitis or PN. Of 
these, 112 were excluded and 327 were enrolled (Figure 1). Patient demographics are shown in Table 3.

Interobserver agreement was calculated for cystitis versus PN $(\kappa=0.84)$; history of previous UTI $(\kappa=$ $0.73)$; antibiotics in the past 3 months $(\kappa=0.63)$; and hospital visit in the last 4 weeks $(\kappa=0.53)$.

Patients with uncomplicated cystitis and $\mathrm{PN}$ had similar median ages of 32 and 31 years, respectively $(p=0.2)$. By comparison, patients presenting with complicated cystitis and PN were older, with median ages of 65.5 and 48.5, years, respectively $(p<0.001$ when compared to the respective uncomplicated diagnosis; see Table 3).

The prevalence of antibiotic use in the preceding 3 months, hospital visit in the last 4 weeks, and UTI in the previous year was similar for patients with uncomplicated cystitis and uncomplicated PN. The first two risk factors were more common in patients with complicated cystitis or complicated PN. A history of UTI in the previous year was most common in the complicated cystitis group (see Table 3).

\section{Urine culture and sensitivities}

Escherichia coli remains the predominant uropathogen across all diagnostic categories (Table 4). Extendedspectrum $\beta$-lactamases (ESBLs) were found in 6.7\% (18 of 269) of E. coli isolates and were more prevalent in PN than in cystitis, irrespective of the presence of complicating factors.
Table 5 shows antibiotic resistance in all organisms by diagnosis and overall. Resistance to ciprofloxacin occurred in $14.7 \%$ of the sample, whereas resistance to trimethoprim-sulfamethoxazole (TMP-SMX) occurred in $27.5 \%$. For uncomplicated cystitis, $24.0 \%$ of urine cultures demonstrated resistance to TMP-SMX, 9.5\% to ciprofloxacin, and $6.8 \%$ to both antibiotics (data not shown). For uncomplicated $\mathrm{PN}$, the rate of resistance was $36.8 \%$ for TMP-SMX, 19.5\% for ciprofloxacin, and $13.8 \%$ for both antibiotics.

Although our numbers of complicated cystitis were low, ciprofloxacin (but not TMP-SMX) resistance was significantly higher in isolates causing complicated cystitis versus uncomplicated cystitis (Table 6). There was no significant difference in the prevalence of TMPSMX or ciprofloxacin-resistant isolates in uncomplicated versus complicated PN.

\section{Risk factors for antibiotic resistance in UTI (cystitis and PN combined)}

Given that it is difficult to differentiate clinically between upper and lower tract urinary infections, cystitis and PN data were combined to evaluate the potential risk factors for antibiotic resistance. The results are not stratified by organism as empirical antibiotic treatment is generally initiated before this information is available.

Only 86 of the 327 charts (26.3\%) had documentation as to whether antibiotics were used in the previous 3 months. Documentation of previous UTI in the past

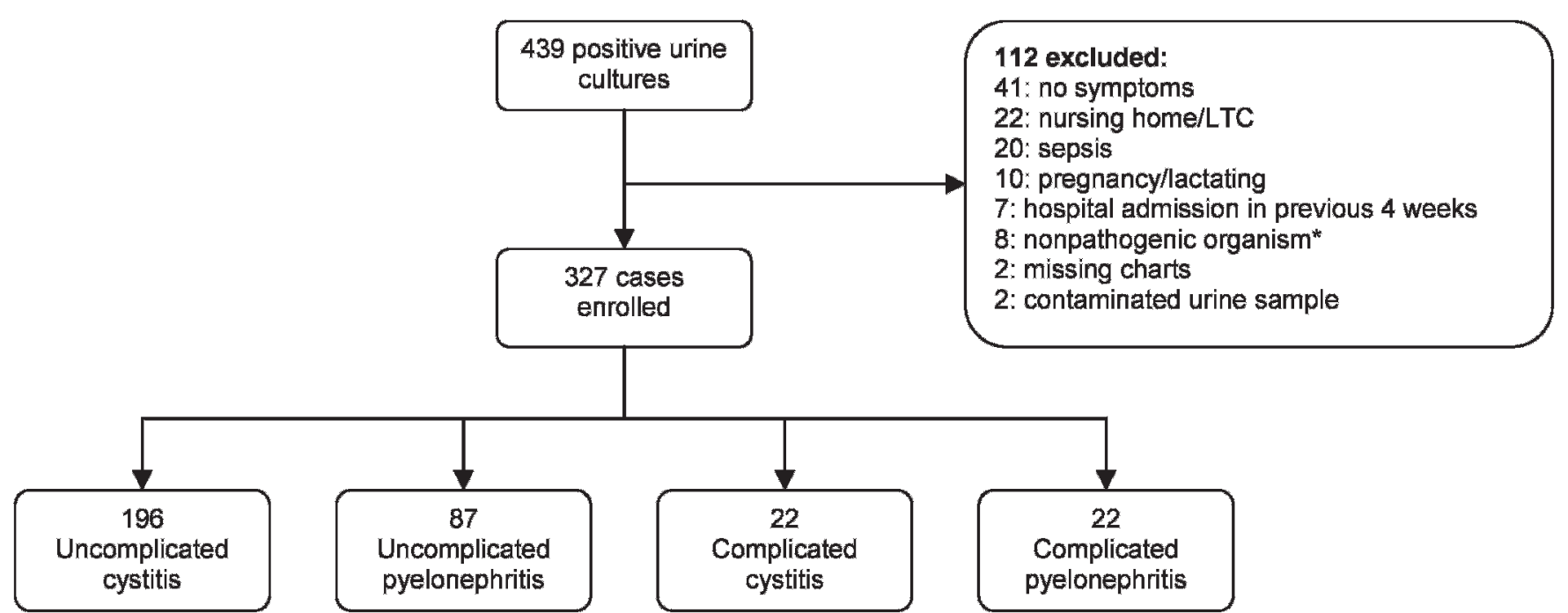

Figure 1. Case flow chart. LTC = Iong-term care. *Included Streptococcus viridans, lactose-fermenting gram-negative bacilli, diphtheroids, and yeast. 


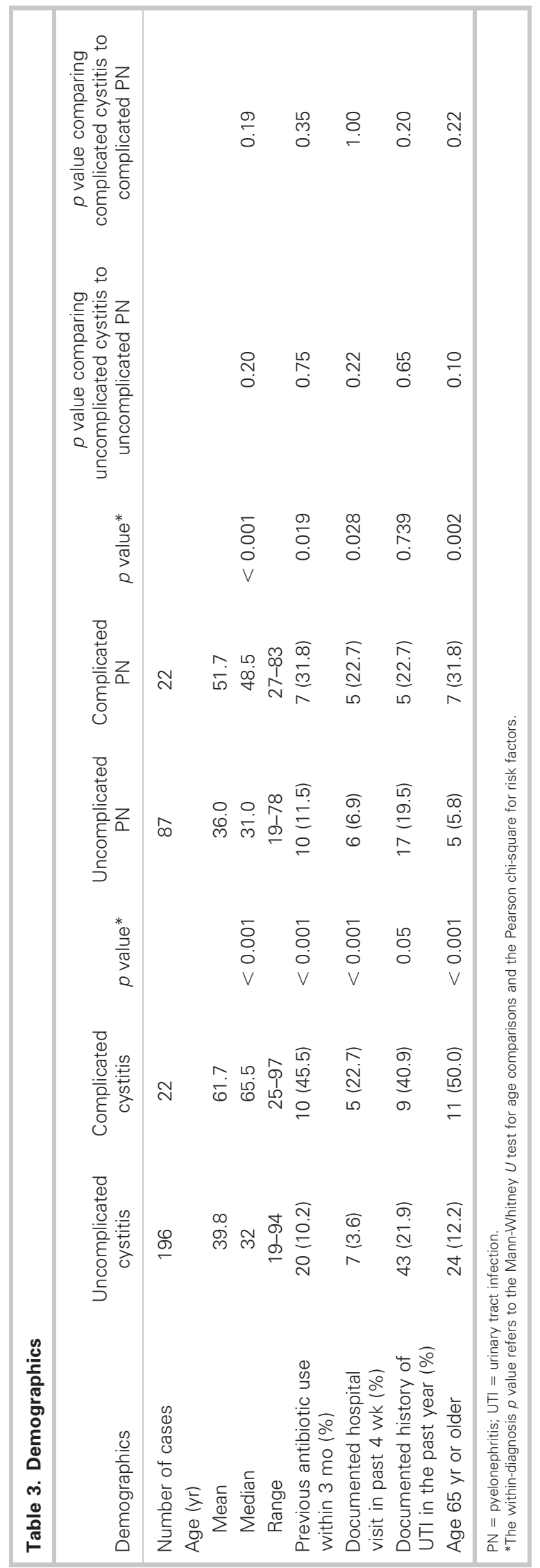

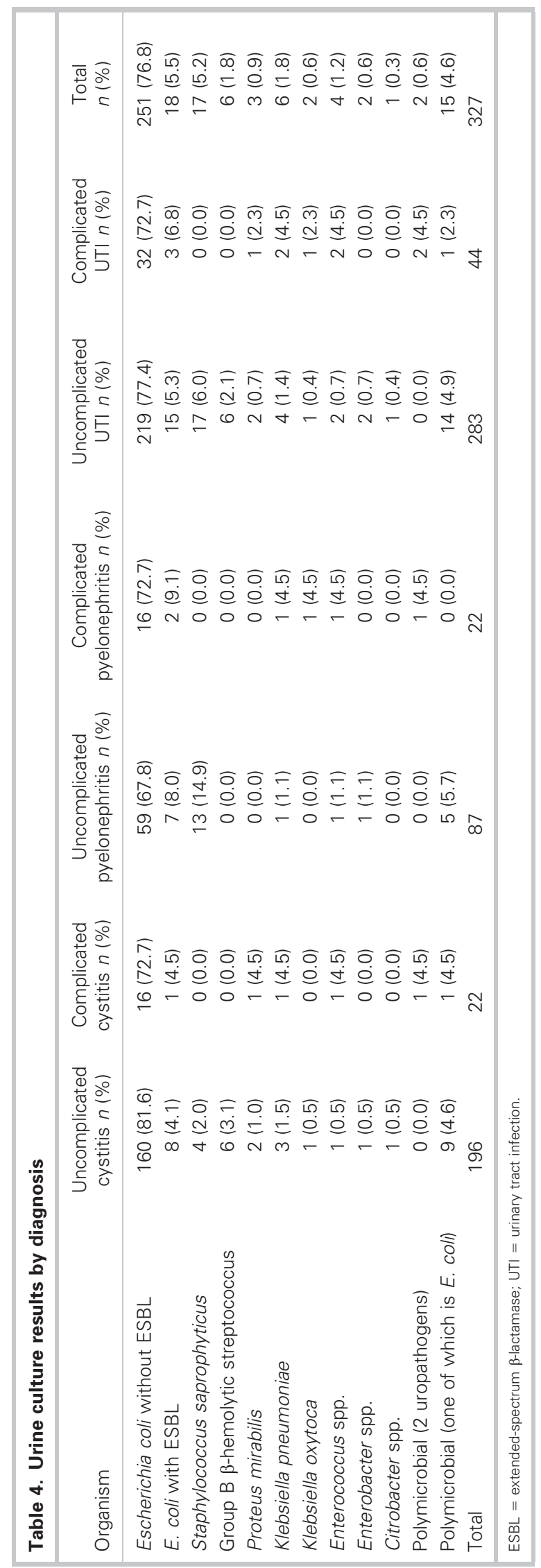




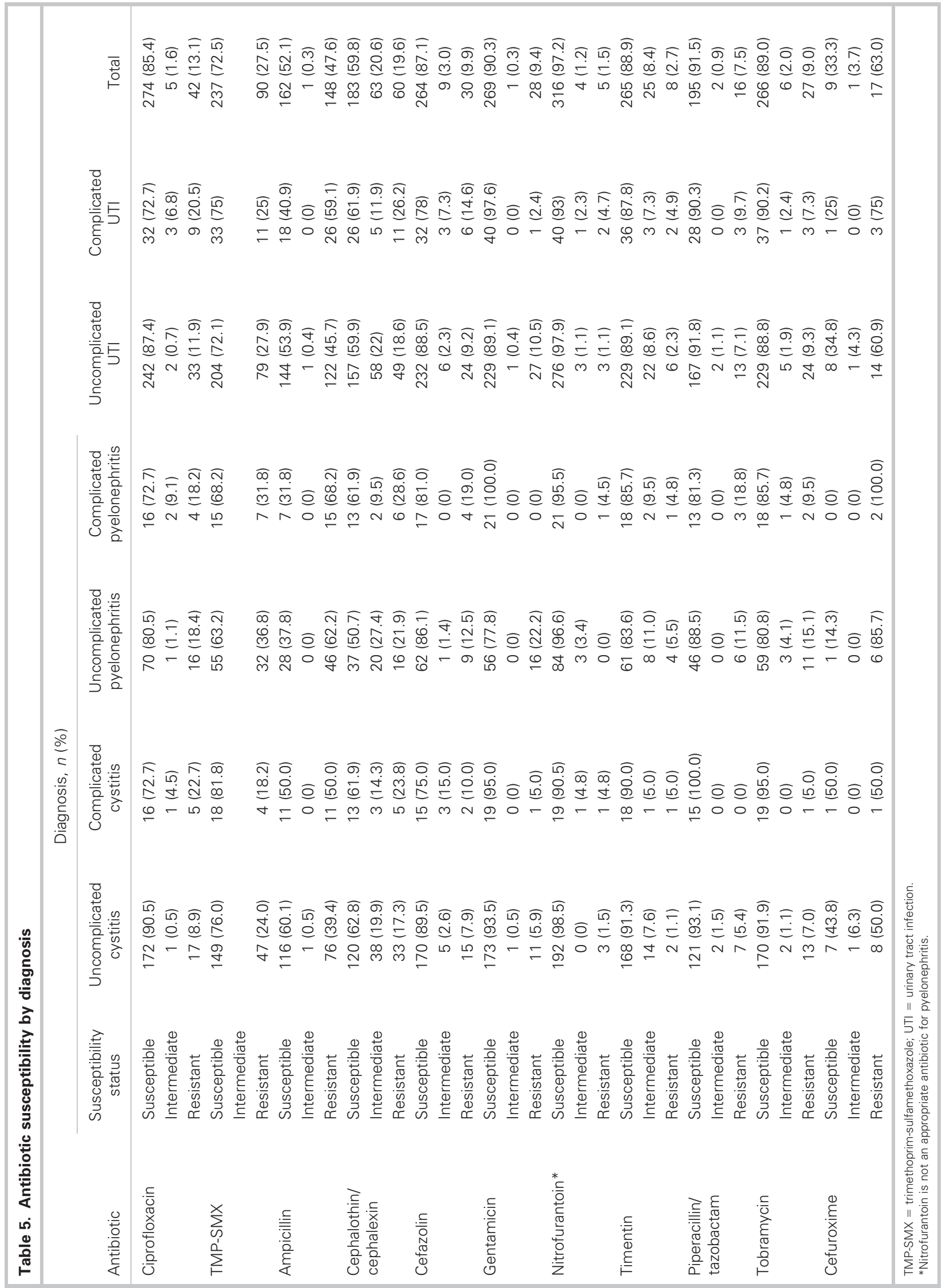


Table 6. Percentage resistance (including intermediate susceptibility) to ciprofloxacin and TMP-SMX by diagnosis

\begin{tabular}{lcc}
\hline & $\begin{array}{c}\text { Ciprofloxacin } \\
\text { resistance }\end{array}$ & $\begin{array}{c}\text { TMP-SMX } \\
\text { resistance }\end{array}$ \\
Uncomplicated cystitis (\%) & 9.5 & 24.00 \\
Complicated cystitis (\%) & 27.3 & 18.20 \\
Fisher exact test, one sided, p value & 0.02 & 0.38 \\
Uncomplicated PN (\%) & 19.5 & 36.8 \\
Complicated PN (\%) & 27.3 & 31.8 \\
Fisher exact test, one sided, p value & 0.30 & 0.43 \\
Uncomplicated UTI (\%) & 12.60 & 27.90 \\
Complicated UTI (\%) & 27.30 & 25.00 \\
Fisher exact test, one sided, p value & 0.01 & 0.42 \\
\hline PN = pyelonephritis; TMP-SMX = trimethoprim-sulfamethoxazole; UTI = urinary tract \\
infection.
\end{tabular}

year was also incomplete, being mentioned in 154 (47\%) charts. Therefore, for our risk factor analysis, we combined all UTIs (cystitis, PN, complicated and uncomplicated). Any antibiotic use in the previous 3 months was a statistically significant risk factor for ciprofloxacin and TMP-SMX resistance (Table 7). A history of UTI and age over 65 were statistically significant risk factors for ciprofloxacin resistance but not TMP-SMX resistance. Due to sparse data, adjusted ORs are not reported.

Further stratification by uncomplicated/complicated UTI did not demonstrate any statistically significant risk factors, although our numbers were small.

\section{DISCUSSION}

With the emergence of antibiotic resistance, the treatment of UTIs has become complex. Neither outpatient laboratory studies that use consecutive midstream samples without patient clinical presentation nor hospital antibiograms can adequately guide management

\begin{tabular}{|c|c|c|c|}
\hline & $\begin{array}{l}\text { Ciprofloxacin } \\
\text { resistance, } n(\%)\end{array}$ & $\begin{array}{c}\text { Ciprofloxacin } \\
\text { sensitivity, } n(\%)\end{array}$ & OR $(95 \% \mathrm{Cl})$ \\
\hline Antibiotic use & $n=23$ & $n=61$ & \\
\hline Have not used antibiotics in the previous $3 \mathrm{mo}$ & $6(26.1)$ & $33(54.1)$ & $3.34(1.16-9.62)$ \\
\hline Have used antibiotics in the previous 3 mo & $17(73.9)$ & $28(45.9)$ & \\
\hline Hospital visit & $n=47$ & $n=274$ & \\
\hline No documented history of hospital visit in the past $4 \mathrm{wk}$ & $41(87.2)$ & $258(94.2)$ & $2.36(0.87-6.38)$ \\
\hline Documented history of hospital visit in the past $4 \mathrm{wk}$ & $6(12.8)$ & $16(5.8)$ & \\
\hline History of UTI & $n=25$ & $n=126$ & \\
\hline No documented history of a UTI in the past year & $8(32.0)$ & $70(55.6)$ & $2.66(1.07-6.61)$ \\
\hline Documented history of UTI in the past year & $17(68.0)$ & $56(44.4)$ & \\
\hline Age & $n=47$ & $n=274$ & \\
\hline$<65 \mathrm{yr}$ & $35(74.5)$ & $241(88.0)$ & $2.50(1.18-5.30)$ \\
\hline \multirow[t]{2}{*}{$\geq 65 \mathrm{yr}$} & $12(25.5)$ & $33(12.0)$ & \\
\hline & $\begin{array}{l}\text { TMP-SMX } \\
\text { resistance }\end{array}$ & $\begin{array}{l}\text { TMP-SMX } \\
\text { sensitivity }\end{array}$ & OR $(95 \% \mathrm{Cl})$ \\
\hline Antibiotic use & $n=29$ & $n=57$ & \\
\hline Have not used antibiotics in the previous $3 \mathrm{mo}$ & $7(24.1)$ & $32(56.1)$ & $4.02(1.48-10.92)$ \\
\hline Have used antibiotics in the previous 3 mo & $22(75.9)$ & $25(43.9)$ & \\
\hline Hospital visit & $n=90$ & $n=237$ & \\
\hline No documented history of hospital visit in the past $4 \mathrm{wk}$ & $83(92.2)$ & 220 (92.8) & $1.09(0.44-2.73)$ \\
\hline Documented history of hospital visit in the past $4 \mathrm{wk}$ & $7(7.8)$ & $17(7.2)$ & \\
\hline History of UTI & $n=46$ & $n=108$ & \\
\hline No documented history of a UTI in the past year & $24(52.2)$ & $56(51.9)$ & $0.99(0.50-1.97)$ \\
\hline Documented history of UTI in the past year & $22(47.8)$ & $52(48.1)$ & \\
\hline Age & $n=90$ & $n=237$ & \\
\hline$<65 y r$ & $76(84.4)$ & $204(86.1)$ & \\
\hline$\geq 65 \mathrm{yr}$ & $14(15.6)$ & 33 (13.9) & $1.14(0.58-2.24)$ \\
\hline
\end{tabular}


of community-acquired UTI. Appropriate treatment should rest on valid information about local resistance rates, ideally derived from community-based isolates linked to presenting symptomatology. Our study sought to describe such a community-based sample.

The uropathogens associated with uncomplicated community-acquired cystitis and $\mathrm{PN}$ are consistent with previous studies, ${ }^{20,21}$ with $E$. coli accounting for $82.7 \%$ of uncomplicated infections followed by Staphylococcus saprophyticus at $6 \%$ and group B streptococcus at $2.1 \%$, and the rest made up of enterococci and other gram-negative rods (see Table 4). The low number of complicated UTIs overall makes it hard to establish a valid trend in terms of their causative uropathogens, but, as expected, Proteus mirabilis and Klebsiella species (pneumoniae and oxytoca), as well as Enterococcus species, were more commonly encountered. ${ }^{13,17}$

The 2010 Infectious Diseases Society of America (IDSA) guidelines on uncomplicated cystitis in women suggest that fluoroquinolones should be avoided unless other options have been exhausted. Nitrofurantoin and TMP-SMX, if resistance prevalence is known to be lower than $20 \%$, are the recommended antimicrobial therapies.22

The high rates of TMP-SMX resistance in this study (24\% for uncomplicated cystitis and $36.8 \%$ for uncomplicated PN) make TMP-SMX an unreliable choice for uncomplicated community-acquired UTI in our ED adult female population. Our results are similar to those of a 2004 study of communityacquired UTIs in adult women in British Columbia, which also found a high TMP-SMX resistance rate $(20.5 \%)$ in isolates growing E. coli. ${ }^{23}$

In British Columbia, there is a well-documented trend of increasing ciprofloxacin resistance in uropathogens, with the rate of resistance increasing from $2.5 \%$ in 1998 to over $20 \%$ in 2007; this correlates with the increased use of fluoroquinolone antibiotics. ${ }^{24}$ In our ED, ciprofloxacin was the most commonly prescribed antibiotic for uncomplicated cystitis during our study period, accounting for $52.7 \%$ of prescriptions (data not shown). This may not be wise, and, when possible, preference should be given to nonfluoroquinolone alternatives, such as nitrofurantoin (see Table 5). However, nitrofurantoin has limited activity against non- $E$. coli urinary pathogens, which are more commonly encountered with complicated cystitis.

The 2010 IDSA guidelines for acute uncomplicated $\mathrm{PN}$ in women also suggest that an oral fluoroquinolone is appropriate outpatient therapy "where the prevalence of community uropathogens to fluoroquinolones is not known to exceed $10 \% .{ }^{\prime 22}$ When the resistance exceeds $10 \%$, expert opinion recommends "an initial one-time long-acting parenteral antimicrobial" in addition to the prescription of oral ciprofloxacin. In our study, resistance to ciprofloxacin for uncomplicated $\mathrm{PN}$ was $19.5 \%$, well above that number. This suggests the need for a 24hour dose of an aminoglycoside or an intravenous dose of ceftriaxone. Unfortunately, the IDSA guidelines make no recommendation as to what fluoroquinolone resistance level mandates altogether an "alternative agent either in conjunction" or as replacement of an oral fluoroquinolone for the outpatient treatment of PN.

Our results of antibiotic susceptibility (see Table 5) for uncomplicated $\mathrm{PN}$ in the face of high prevalence of in vitro ciprofloxacin resistance do not allow us to offer an oral antibiotic alternative. Too few isolates were tested against cefuroxime to draw any valid conclusion, and nitrofurantoin is not acceptable for PN because it does not achieve sufficient serum levels.

Any antibiotic use in the previous 3 months was the only significant risk factor for both TMP-SMX and ciprofloxacin resistance in UTI. This finding is in keeping with previous studies of community-acquired cystitis, ${ }^{25,26}$ although our results are based on all UTIs combined. In our ED, patients with UTI who had been on antibiotics in the last 3 months were more than three times as likely to have an isolate resistant to ciprofloxacin than those who had not taken any recent antibiotic. Similarly, women were four times as likely to have an isolate resistant to TMP-SMX than those who had not taken any recent antibiotic. Patients with a history of UTI within the previous year were 2.7 times more likely and those over age 65 were 2.5 times more likely to have a urinary isolate resistant to ciprofloxacin.

\section{Limitations}

Several limitations should be taken into account when evaluating our results. Medical record reviewers were not blinded to the study hypothesis; however, strict adherence to recommendations for chart reviews ${ }^{18,19}$ helped minimize potential bias in this regard.

Common pitfalls of medical record reviews (e.g., illegibility, incomplete documentation) may account for the low frequency of potential risk factors for antibiotic resistance and, ultimately, lack of significance. The small number of risk factors documented meant that 
stratifying by type of diagnosis (complicated and uncomplicated) was not feasible. A clinician will not always have the patient's full history available to assign a diagnosis of uncomplicated or complicated UTI, so interpreting the risk factors for all UTIs combined is clinically relevant.

There is a lack of standardization in the literature of various UTI-related definitions including "positive urine culture," making comparisons difficult. Our definitions (see Table 1) are consistent with those in the literature and have face validity. Our criteria for both cystitis and PN are symptom based, which would exclude paraplegics (4 cases were excluded), patients with altered sensation ( 2 multiple sclerosis patients), and the elderly with UTI presenting with delirium, general weakness, and vague symptoms (28 cases). These were among the 41 asymptomatic patients excluded in Figure 1. Unlike some studies, ${ }^{6,27}$ we did not require a documented fever as a criterion for PN. We made this decision because immunosuppressed patients, the elderly, and patients who take antipyretics prior to their $\mathrm{ED}$ visit may have $\mathrm{PN}$ without a documented fever. The low numbers of patients with complicated UTI in our study limit our ability to draw conclusions in this category. These low numbers may be due to our exclusion criteria.

We took a conservative approach and classified organisms with intermediate antibiotic resistance as resistant. We recognize that patients may improve despite being treated with an antibiotic to which the organism has only intermediate resistance. However, we found that any antibiotic use in the prior 3 months was a risk factor for both ciprofloxacin and TMPSMX resistance regardless of whether intermediate results were classified as resistant or not. Similarly, a history of UTI in the last year and age over 65 remained risk factors for ciprofloxacin regardless of the classification. These findings increase our confidence in our conclusions.

Our findings are limited to the population we studied, that is, adult women, age 19 or greater, nonpregnant and nonlactating, who presented to our tertiary care Vancouver ED with community associated-UTI and had a positive urine culture. With ED overcrowding, nurse-initiated investigations at our institution have led to urine cultures being sent on most UTI patients; however, patients with UTIs without a urine culture were not included or tracked to ensure that they were similar to our study subjects.
Finally and most importantly, our study was not an outcome study; thus, we can only comment on in vitro resistance to antibiotic or microbiologic failure rather than clinical failure.

\section{CONCLUSION}

E. coli was the predominant uropathogen across all UTI categories in the adult nonpregnant women with community-acquired UTIs who presented to our ED in 2008. Fifteen percent of all UTIs were caused by isolates resistant to ciprofloxacin and $27.5 \%$ by those resistant to TMP-SMX. Any antibiotic use in the previous 3 months was a significant risk factor for both ciprofloxacin and TMP-SMX uropathogen resistance. Being 65 years of age or older and having had a history of UTI in the previous year were risk factors only for ciprofloxacin resistance. Future prospective outcome studies, with emphasis on clinical cure rather than microbiologic cure, as well as patient-specific risk factors associated with antibiotic resistance, are needed to guide UTI management.

Competing interests: Dr. Brubacher's research is supported by a scholar award from the Michael Smith Foundation for Health Research.

\section{REFERENCES}

1. Mazzulli T. Resistance trends in urinary tract pathogens and their impact on management. Infect Dis Microbiol Rounds 2002;2:5.

2. Aubin C. Does this woman have an acute uncomplicated urinary tract infection? Ann Emerg Med 2007;49:106-8, doi:10.1016/j.annemergmed.2006.09.022.

3. Seupal R, McDowell C, Bassett R. Urinary tract infection. In: Rowe B, editor. Evidence-based emergency medicine. Chichester (UK): Blackwell Publishing; 2009. p. 412-9.

4. Kunin C. Urinary tract infections: detection, prevention, and management. Baltimore: Williams \& Wilkins; 1997.

5. Ikaheimo R, Siitonen A, Heiskanen T, et al. Recurrence of urinary tract infection in a primary care setting: analysis of a 1-year follow-up of 179 women. Clin Infect Dis 1996;22:91-9, doi:10.1093/clinids/22.1.91.

6. Talan DA, Stamm WE, Hooton TM, et al. Comparison of ciprofloxacin (7 days) and trimethoprim-sulfamethoxazole (14 days) for acute uncomplicated pyelonephritis in women. FAMA 2000;283:1583-90, doi:10.1001/jama.283.12.1583.

7. Raz R, Chazan B, Kennes Y, et al. Israeli Urinary Tract Infection Group. Empiric use of trimethoprim-sulfamethoxazole (TMP-SMX) in the treatment of women with uncomplicated urinary tract infections, in a geographical area with a high prevalence of TMP-SMX resistant uropathogens. Clin Infect Dis 2002;34:1165-9, doi:10.1086/ $\underline{339812}$. 
8. McNulty CAM, Richards J, Livermore DM, et al. Clinical relevance of laboratory-reported antibiotic resistance in acute uncomplicated urinary tract infection in primary care. $\mathcal{F}$ Antimicrob Chemother 2006;58:1000-8, doi:10.1093/jac/dkl368.

9. Alam MF, Cohen D, Butler C, et al. The additional costs of antibiotics and re-consultations for antibiotic-resistant Escherichia coli urinary tract infections managed in general practice. Int 7 Antimicrob Agents 2009;33:255-7, doi:10.1016/ j.ijantimicag.2008.08.027.

10. Karlowsky JA, Kelly LJ, Thornsberry C, et al. Trends in antimicrobial resistance among urinary tract infection isolates of Escherichia coli from female outpatients in the United States. Antimicrob Agents Chemother 2002;46:2540-5, doi:10.1128/AAC.46.8.2540-2545.2002.

11. Zhanel GG, Hisanaga TL, Laing NM, et al. Antibiotic resistance in Escherichia coli outpatient urinary isolates: final results from the North American Urinary Tract Infection Collaborative Alliance (NAUTICA). Int 7 Antimicrob Agents 2006;27:468-75, doi:10.1016/j.ijantimicag. 2006.02.009.

12. Drekonja DM, Johnson JR. Urinary tract infections. Prim Care 2008;35:345-67, doi:10.1016/j.pop.2008.01.001.

13. Abrahamian FM, Moran GJ, Talan DA. Urinary tract infections in the emergency department. Infect Dis Clin North Am 2008;22:73-87, doi:10.1016/j.idc.2007.10.002.

14. Nicolle LE. Epidemiology of urinary tract infections. Clin Microbiol Newsl 2002;24:135-40, doi:10.1016/S0196-4399 (02)80035-6.

15. Hooton TM, Besser R, Foxman B, et al. Acute uncomplicated cystitis in an era of increasing antibiotic resistance: a proposed approach to empirical therapy. Clin Infect Dis 2004; 39:75-80, doi:10.1086/422145.

16. Miller LG, Tang AW. Treatment of uncomplicated urinary tract infections in an era of increasing antimicrobial resistance. Mayo Clin Proc 2004;79:1048, doi:10.4065/79.8.1048.

17. Carson C, Naber KG. Role of fluoroquinolones in the treatment of serious bacterial urinary tract infections. Drugs 2004;64:1359-73, doi:10.2165/00003495-200464120-00007.

18. Gilbert EH, Lowenstein SR, Koziol-McLain J, et al. Chart reviews in emergency medicine research: where are the methods? Ann Emerg Med 1996;27:305-8, doi:10.1016/ S0196-0644(96)70264-0.

19. Worster A, Bledsoe RD, Cleve P, et al. Reassessing the methods of medical record review studies in emergency medicine research. Ann Emerg Med 2005;45:448-51, doi: 10.1016/j.annemergmed.2004.11.021.

20. Gupta K, Hooton TM, Stamm WE. Increasing antimicrobial resistance and the management of uncomplicated community-acquired urinary tract infections. Ann Intern Med 2001;135:41-50.

21. Wagenlehner FME, Naber KG. Suitable antibacterial substances for the treatment of urinary tract infections. Antiinfect Agents Med Chem 2006;5:309-29.

22. Gupta K, Hooton TM, Naber KG, et al. International clinical practice guidelines for the treatment of acute uncomplicated cystitis and pyelonephritis in women: a 2010 update by the Infectious Diseases Society of America and the European Society for Microbiology and Infectious Diseases. Clin Infect Dis 2011;52:e103-20, doi:10.1093/cid/ciq257.
23. McIsaac WJ, Mazzulli T, Permaul J, et al. Communityacquired antibiotic resistance in urinary isolates from adult women in Canada. Can 7 Infect Dis Med Microbiol 2006;17: $337-40$.

24. Patrick DM, Hutchinson J. Antibiotic use and population ecology: how you can reduce your "resistance footprint." CMA尹 2009;180:416-21.

25. Killgore KM, March KL, Guglielmo BJ. Risk factors for community-acquired ciprofloxacin-resistant Escherichia coli urinary tract infection. Ann Pharmacother 2004;38:1148-52.

26. Brown PD, Freeman A, Foxman B. Prevalence and predictors of trimethoprim-sulfamethoxazole resistance among uropathogenic Escherichia coli isolates in Michigan. Clin Infect Dis 2002;34:1061-6, doi:10.1086/339491.

27. Talan DA, Krishnadasan A, Abrahamian FM, et al. EMERGEncy ID NET Study Group. Prevalence and risk factor analysis of trimethoprim-sulfamethoxazole and fluoroquinolone-resistant Escherichia coli infection among emergency department patients with pyelonephritis. Clin Infect Dis 2008;47:1150-8, doi:10.1086/592250.

\begin{tabular}{|l}
\hline Appendix 1. ICD-9 and ICD-10-CA codes \\
ICD-9 codes \\
590.0 Chronic pyelonephritis \\
590.1 Acute pyelonephritis \\
590.2 Renal and perinephric abscess \\
590.3 Pyeloureteritis cystica \\
590.8 Other pyelonephritis or pyonephrosis, not specified as \\
acute or chronic \\
590.80 Pyelonephritis, unspecified \\
590.81 Pyelitis or pyelonephritis in diseases classified \\
elsewhere \\
590.9 Infection of kidney, unspecified \\
595 Cystitis \\
595.0 Acute cystitis \\
595.2 Other chronic cystitis \\
595.4 Cystitis in diseases classified elsewhere \\
595.8 Other specified types of cystitis \\
595.9 Cystitis, unspecified \\
599.0 Urinary tract infection, site not specified \\
599.7 Hematuria \\
599.9 Unspecified disorder of urethra and urinary tract \\
ICD-10-CA codes \\
N30.0 Acute cystitis \\
N30.2 Other chronic cystitis \\
N30.8 Other cystitis \\
N30.9 Cystitis, unspecified \\
N39.8 Other specified disorders of the urinary system \\
N39.9 Disorder of the urinary system, unspecified \\
R31.0 Gross hematuria \\
R31.1 Microscopic hematuria \\
\end{tabular}




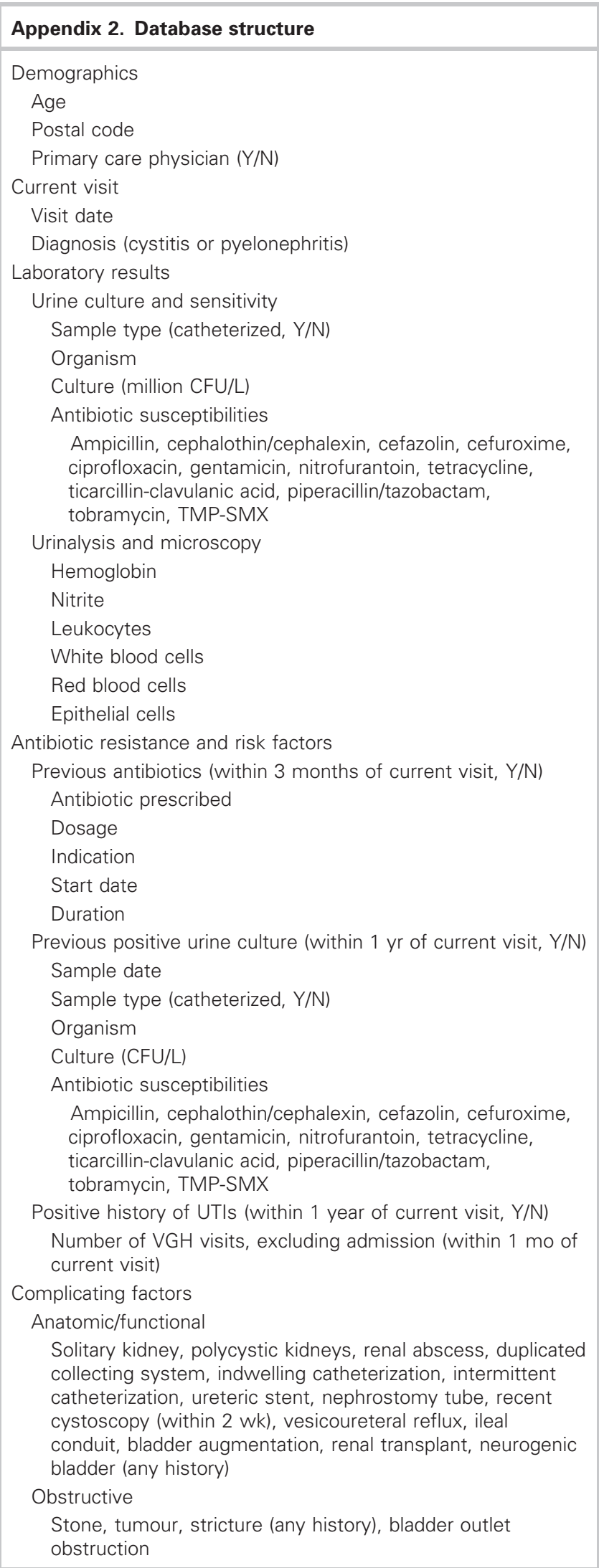

\begin{tabular}{l} 
Appendix 2 . Continued \\
Medical conditions \\
IDDM, NIDDM, renal failure (SCr $>200$ mol/L, GFR $<$ \\
$30 \mathrm{~mL} /$ min), hepatic failure, immunosuppression, \\
corticosteroid use, HIV, active cancer (within 6 mo) \\
Antibiotic allergies \\
Penicillins, cephalosporins, sulphas, fluoroquinolones, \\
nitrofurantoin \\
Disposition \\
Admitted \\
Admission date \\
Admission length \\
Discharged \\
Antibiotic prescribed \\
Dosage \\
Duration \\
IV fast track \\
Antibiotic prescribed \\
Dosage \\
Duration \\
Additional UTIs \\
Additional cystitis cases (2008) \\
Additional pyelonephritis cases (2008) \\
Return visit \\
Visit date \\
Reason(s) for return \\
Disposition \\
Admitted \\
$\quad$ Admission date \\
Admission length \\
DFischarged \\
dependent diabetes mellitus; IV = intravenous; NIDDM = non-insulin-dependent \\
UTI = urinary tract infection; VGH = Vancouver General Hospital. \\
\hline
\end{tabular}

